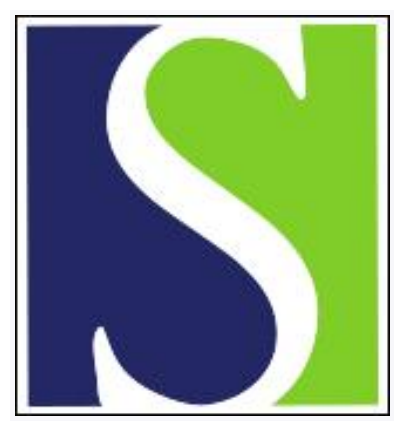

Scand J Work Environ Health 1999;25(6):537-541

https://doi.org/10.5271/sjweh.477

Issue date: Dec 1999

Progress and potential of genetic susceptibility to environmental toxicants

by Haugen $A$

Key terms: gene-environment interaction; microchip; molecular epidemiology; polymorphism; single-nucleotide polymorpism

This article in PubMed: www.ncbi.nlm.nih.gov/pubmed/10884150

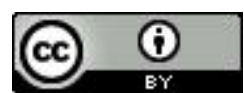




\title{
Progress and potential of genetic susceptibility to environmental toxicants
} by Aage Haugen, $P h D^{1}$

\begin{abstract}
Haugen A. Progress and potential of genetic susceptibility to environmental toxicants. Scand J Work Environ Health 1999;25(6, special issue): $537-540$.

Gene-environment interactions are thought to be critical for such multifactorial diseases as cancer, diabetes, heart disease, asthma, and some neurological disorders. The genetic constitution of an individual (genotype) may influence the risk of disease of a person exposed to environmental or occupational insults. Major advances will occur in the coming years with respect to the identification of the genetic and molecular causes of susceptibility to common diseases. In these studies microarrays and chip technology are rapidly becoming central in the detection of mutations and polymorphisms and in functional genomics. These rapid advances in genetics present new and complex ethical issues for both the individual and society.
\end{abstract}

Key terms gene-environment interaction, microchips, molecular epidemiology, polymorphism, single-nucleotide polymorphisms.

Since all people have the same basic set of genes, why does one heavy smoker live to celebrate his 100th birthday while another develops cancer or heart disease at the age of 50 ? What underlies the interindividual variation in disease risk and responses to the environment? Many factors are involved, but of primary importance in disease development is the interaction between genetic risk factors and environmental exposures (figure 1). Understanding of the genotype-environment interaction in disease development may lead to a better understanding of disease pathogenesis and should lead to improved preventive measures and therapeutic intervention. This understanding will be improved as the technologies unravel the human genome. The ultimate goal of the Human Genome Project (HUGO) is to map and sequence the 3 billion DNA (deoxyribonucleic acid) bases of all human genes and to increase our knowledge of gene function. Due to the recent development in molecular genetic technologies the project is making good progress, and the sequence of the $70000-100000$ total human genes is presently scheduled for completion no later than in 2003. This vast international undertaking will revolutionize modern biology in the 21 th century beyond what is currently conceivable. The biomedical research community is already beginning to formulate strategies for the postgenome era.

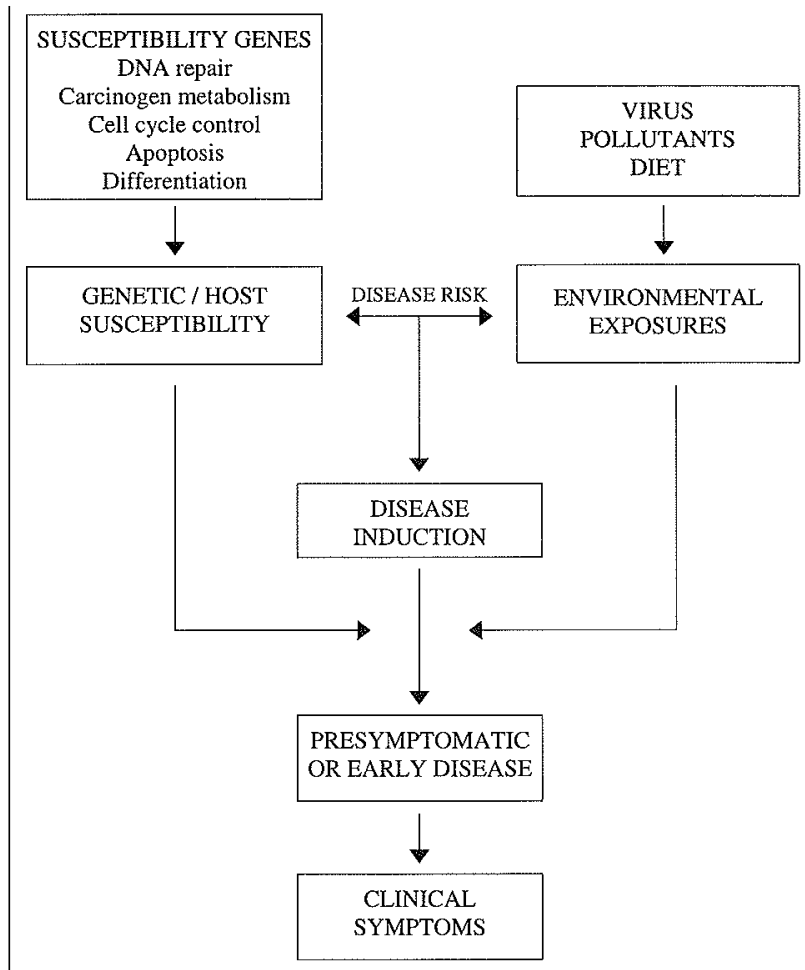

Figure 1. Gene-environment interactions: towards a molecular explanation of individual variations in responsiveness to chemical agents.

Reprint requests to: Dr Aage Haugen, Department of Toxicology, National Institute of Occupational Health, PO Box 8149 Dep, N-0033 Oslo, Norway. [E-mail: age.haugen@stami.no] 


\section{Genes are only part of the complex picture}

Many of the substances humans are exposed to pose a health risk. Thus there is a need to come up with a strategy to understand the mechanisms by which these compounds impair human health and also to identify (sub)populations or persons predisposed to develop the adverse effects. It is now feasible to approach these challenging questions and the identifications of genes that contribute to increased sensitivity to toxins in the environment.

Several studies have clearly demonstrated that genes may have a strong influence on the development of disease. Over the last $10-15$ years the identification of genes causing monogenic (single-gene) disorders has been given much attention. So far nearly all common genetic diseases and several rare ones have been related to defective gene(s), for example the gene responsible for cystic fibrosis, Duchenne muscular dystrophy, Huntington's disease, and others. Mutations in the 2 copies of the cystic fibrosis gene are fully penetrant: the person develops the disease, and the outcome is not modified by environmental factors. In addition, phenotype variation between persons carrying the same mutation in a high-penetrance gene has been described. Monogenic disorders usually affect only relatively few people (1). Recently, the emphasis has been shifted towards the identification of genes involved in what is known as polygenic non-Mendelian disorders. For many of these common complex multifactorial diseases, such as cancer, asthma, diabetes, heart disease and some forms of mental illness, environmental factors are important in determining whether susceptible people develop the disease. In cancer, for instance, the familial forms of hereditary predisposition to cancer are attributable to dominantly inherited germ-line mutations (such as RB1, TP53, WT1, or $A P C$ ) that are usually rare and produce high relative risks for one or more forms of cancer. Environment plays a minor role in the causation of these cancers. In contrast, there are predispositions in which several genes and exposure work interactively over decades with much lower relative risks and in which the environment plays a major role in disease development. For instance, up to $80 \%$ of human cancers are thought to arise as a consequence of exposure to environmental agents. The cancer susceptibility (predisposition) genes with less penetrance are involved in common "sporadic" cancers affecting a large segment of the population. Generally, alleles associated with common multifactorial disorders tend to occur frequently (more than 10\%) in the healthy population. Examples are the polymorphic carcinogen-metabolizing genes (2) and the genes for apolipoprotein $\mathrm{E}$ in Alzheimer's disease (3). Defining the influence of each of the multiple genes in the causation of our most common disease and their interactions with environmental elements is a new challenge and is one of the main targets of biomedical research. The goal is to separate the factors involved - to understand the contribution of the genes so we can better understand the contribution of the environment.

\section{Individual variation in responsiveness to environmental agents}

Individual responses to environmental factors may be due to the effects of a variation known as genetic polymorphism (ie, common genetic variants that occur with a frequency of $1 \%$ or more). The change may alter slightly the composition of the protein encoded by the gene. Variation in DNA sequences can have a major impact on how humans respond to environmental insults even though more than $99 \%$ of human DNA sequences are the same in the population. To date relatively few susceptibility genes that modify a person's risk of cancer and other diseases have been explored with respect to genetic polymorphism. Recent investigations have focused on the role of polymorphism in biotransformation pathways. Several studies have shown that the polymorphism of cytochrome P450 (CYP) isoenzymes, glutathione S-transferases (GST) and N-acetyl-transferase (NAT) enzymes cause defects in the catalytic function of mutated enzymes. A variety of potentially carcinogenic and toxic compounds are metabolized by phase I enzymes, including the CYP system, the epoxide hydrolase family, and phase II enzymes such as GST, NAT, NADPH:quinone oxidoreductase, sulfotransferases, and others. These enzymes represent the first line of defense against environmental agents. As the highly reactive products of phase I enzymes react with DNA, RNA (ribonucleic acid) or proteins, it is clear that the expression of phase I and II enzymes must be well-coordinated. In relation to cancer susceptibility, 3 supergene families have attracted recent interest: $C Y P, G S T$, and $N A T$ genes.

In the past, several molecular-epidemiologic studies have attempted to identify gene-environment interaction in the cancer process. Genetic polymorphisms in xenobiotic metabolizing genes have been found to increase cancer risk for many different organ sites. So far most of the studies have been a single genotype approach, and the studies have been relatively small. The data obtained for some of the genotype analyses are conflicting, and therefore they may at least be interpreted as indicating that the effect of this single polymorphism is low. There are many reviews on the topic $(2,5,6)$. Most of the studies have been on the molecular epidemiology of lung cancer, and several gene-environment interactions for lung cancer risk have been demonstrated whereby genetic polymorphisms in carcinogen-metabolizing genes have been 
shown to modify the effect of the carcinogen exposure on cancer risk, DNA-adduct levels, and mutations (7). Examples are Japanese studies in which the genetic role for the metabolism of carcinogens by $C Y P 1 A 1$ and GSTMI has been established, showing a high relative risk at a low dose level of cigarette smoking for people with susceptible genotypes (8). Studies on the combination of risk genotypes in lung cancer risk show that, among squamous-cell carcinoma patients, those with the CYP1A1 MspI genotype combined with the GSTMI deficient null genotype were at remarkably higher risk of developing this type of tumor (9).

Another approach has been the identification of markers that are closer to the primary effects of susceptibility genes than to clinical symptoms. For instance, the impact of the different genotypes on intermediate end points (ie, the formation of DNA adducts). In the lung, for instance, studies have shown considerable interindividual variation in carcinogen-DNA binding under equivalent conditions of exposure: $50 \%$ of lung cancer patients have smoked between 15-20 cigarettes per day, but the variation in adduct levels in the group was 25 -fold (10). Several factors can influence the number of DNA adducts. Several laboratories have been able to show that carcinogen-metabolizing genes can modify the level of adduction in vivo. For example, lung PAH-DNA adduct levels $(\mathrm{PAH}=$ polycyclic aromatic hydrocarbons) have been related to CYP1A1, GSTM1, and GSTP1 polymorphism $(11,12)$. Furthermore, the different genotype distribution found in patients with transversion and transition mutations in the TP53 and $k$-ras genes also supports the results: a prevalence of transversions at GC in TP53 and $k$-ras genes was found among patients with the GSTMI null genotype (12). Thus the results seem to indicate that genetic factors are involved in the disposition to high levels of adducts and mutational types. The number of cigarettes smoked per day had only a low significance for the variation in adduct levels. It can therefore be assumed that some people are predisposed to high adduct levels without regard to their daily smoking dose. Statistically these people have an earlier onset of lung cancer than people with low adduct levels $(10,13)$.

\section{New goals focusing on individual variations in the human genome}

So far research efforts have focused mostly on the mechanisms of carcinogen metabolism. One can anticipate that major advances will occur in this field in the future. This area will benefit directly from the current development in microchips array technologies and the data base on DNA sequence information. These efforts of characterizing the variation in genetic material are now broadening to cover genes involved in DNA repair, DNA replication, and cell cycle control. The Environmental Genome Project (EGP) has been initiated by the National Institute for Environmental Health and Safety (NIEHS). The strategy is to characterize genes (involving 200 genes and 1000 persons), coding for proteins critical in the bodily response to environmental agents, and to relate these differences to the susceptibility of humans to chemicals in the environment (14). In this project the strategy is to use the large-scale technology developed in HUGO to identify the allelic variants of environmental disease susceptibility genes. The next stage is to develop a data base of known polymorphisms for these genes and use microchip technology to investigate geneenvironment interaction in disease etiology. This strategy has a great power to detect genes involved in polygenic disease and also provides a platform for studies on individual responses to environmental toxins. As significant variants are identified, exposed people can be classified by risk characteristics. This approach will increase the sensitivity of molecular epidemiologic studies and environmental components of disease risk will be identified.

Microarray and DNA chip technology are well suited for DNA sequencing analysis on a genomic scale and gene-expression monitoring. Microarrays contain sequences representing the mRNA of an expressed gene applied at high density to a substrate. DNA chips contain short pieces of DNA sequences (oligonucleotides), each with a known sequence spotted on a substrate. The microarrays or chips are rapidly becoming central in functional genomics and in the rapid detection of mutations and polymorphisms. This technology will also have wide applications in toxicology (toxicogenomics) (1518).

Methods are being developed to detect different types of variation, particularly the most common type called single-nucleotide polymorphisms (SNP). This type of variation is believed to account for at least $90 \%$ of human genetic diversity. SNP maps may be very useful in the identification of candidate susceptibility genes associated with complex diseases and to explain how genetic variation predisposes people to certain diseases by stratifying patient groups $(19,20)$. SNP occurs in about every 1000 bases in the genome. No one really knows how many SNP the genetic code contains or how many are important. Several hundred thousand SNP will be discovered in the near future in ongoing research programs, enough to get a start in the understanding of human diversity and how various SNP interact with one another and predispose people to common diseases (http:// www.ncbi.nlm.gov/SNP/). Eventually the major population variants will be identified, and instruments will be built to allow the genotyping of people for these variants. Expression monitoring data coupled with exposure 
data may also be useful biomarkers in studies of adverse effects of chemical exposure in humans.

\section{Concluding remarks}

In the future, individual genetic susceptibility tests may be undertaken as knowledge of the genetic basis of common diseases becomes better established. People identified as being at high risk for common diseases could adjust their diet, life-style, and other risk factors to reduce the risk of developing the disorder. If we give people information about their risk, at least they will have an increased ability to initiate environmental intervention by avoiding certain exposures.

However, the rapid advances in genetics and its implications present new and complex ethical and policy issues for both individual persons and society. There is a possibility to misuse genetic data in several ways. Several factions of society are interested in such information: employers, insurers, and courts, for example. There is widespread concern about discrimination against people with genetic predisposition to disease and also about the possibility that test results may be misinterpreted. A continuing challenge for policy makers is to safequard the privacy of the individual.

\section{References}

1. Ponder B. Genetic testing for cancer risk. Science 1997;278:1050-54.

2. International Agency for Research on Cancer (IARC). Metabolic polymorphisms and susceptibility to cancer. Lyon: IARC, 1999. IARC scientific publication, no 148.

3. Blacker D, Tanzi RE. The genetics of Alzheimer disease: current status and future prospects. Arch Neurol 1998; 55:294-96.

4. Wormhoudt LW, Commandeur JNM, Vermeuten NPE. Genetic polymorphism of human $\mathrm{N}$-acetyltransferase, cytochrome P450, glutathione-S-transferase, and epoxide hydrolase enzymes: relevance to xenobiotic metabolism and toxicity. Crit Rev Toxicol 1999;29:59-124.

5. Perera FP. Environment and cancer: who are susceptible? Science 1997;278:1068-73.
6. Rebbeck TR. Molecular epidemiology of the human glutathione S-transferase genotypes GSTM1 and GSTT1 in cancer susceptibility. Cancer Epidemiol Biomarkers Prev 1997; 6:733-43.

7. Spivac SD, Fasco MJ, Walker VE, Kaminsky LS. The molecular epidemiology of lung cancer. Crit Rev Toxocol 1997; $27: 319-65$.

8. Okada T, Kawashima K, Fukushi S, Minakuchi T, Nishimura S. Association between a cytochrome P450 CYP1A1 genotype and incidence of lung cancer. Pharmacogen 1995;4:33340.

9. Kihara M, Noda K. Risk of smoking for squamous and small cell carcinomas of the lung modulated by combination of CYP1A1 and GSTM1 gene polymorphisms in a Japanese population. Carcinogenesis 1995;16:2331-36.

10. Ryberg D, Hewer A, Phillips DH, Haugen A. Different susceptibility to smoking-induced DNA damage among male and female lung cancer patients. Cancer Res 1994;54:5801—03.

11. Rojas M, Alexandrov K, Cascorbi I, Brockmöller J, Likhachev A, Pozharisski K, et al. High benzo(a)pyrene diol-epoxide DNA adduct levels in lung and blood cells from individuals with combined CYP1A1 MspI/MspI-GSTM $1 * 0 / * 0$ genotypes. Pharmacogen 1998;8:109-18

12. Ryberg D, Skaug V, Hewer A, Phillips DH, Harries LW, Wolf CR, et al. Genotypes of glutathione transferase M1 and P1 and their significance for lung DNA adduct levels and cancer risk. Carcinogenesis 1997;18:1285-89.

13. Mollerup S, Ryberg D, Hewer A, Phillips DH, Haugen A. Sex differences in lung CYP1A1 expression and DNA adduct levels among lung cancer patients. Cancer Res 1999; 59:3317-20.

14. Kaiser J. Environment institute lays plans for gene hunt. Science 1997;278:569-70

15. DeRisi J, Penland L, Brown PO, Bittner ML, Meltzer PS, Ray $\mathrm{M}$, et al. Use of cDNA microassay to analyse gene expression patterns in human cancer. Nat Genet 1996;14:457-60.

16. Heller RA, Schena M, Chai A, Shalon D, Bedilion T, Gilmore $\mathbf{J}$, et al. Discovery and analysis of inflammatory diseaserelated genes using cDNA microarrays. Proc Natl Acad Sci USA 1997;94:2150-55.

17. Brown PO, Botstein D. Exploring the new world of the genome with DNA arrays. Nat Genet 1999;21 suppl:33-7.

18. Nuwaysir EF, Bittner M, Trent J, Barret JC, Afshari CA. Microarrays and toxicology: the advent of toxicogenomics. Mol Carcinog 1999;24:153-9.

19. Halushka MK, Fan JB, Bentley K, Hsie L, Shen N, Weder A, et al. Patterns of single-nucleotide polymorphisms in candidate genes for blood-pressure homeostasis. Nat Genet 1999:22:239-47.

20. Cargill M, Altshuler D, Ireland J, Sklar P, Ardlie K, Patil N, et al. Characterization of single-nucleotide polymorphisms in coding regions of human genes. Nat Genet 1999;22:231-38. 